ด

columns start had been made in implementing its recommendations but that no health area had put all its recommendations into practice.

The authors of the present report are encouraged by what has been achieved and remain convinced of the importance of the original document's two key recommendations. An incremental approach is advocated, with the appointment of two key players: at the commis sioning level (primary care trusts or their equivalent), a named person responsible for planning, and a consultant clinician to act as a focus for referrals, initially with two programmed activities or sessional equivalents. An old age psychiatrist is well-placed to undertake the clinical role.

After these appointments have been made, the rudiments of the local service are created. At all stages, coordination and networking with people already in volved with younger people with dementia is important; the composition and evolution of the new service will depend on existing local services and facilities.

Both organisations remain committed to these principles and consider that it is timely to publish this second edition of the policy paper so that the momentum will be maintained. In this way this disadvantaged group of patients will have a modern dedicated service to meet their special needs.

\section{Safety for Psychiatrists}

Council Report CR134, January 2006, Royal College of Psychiatrists, f7.50, $32 \mathrm{pp}$

Despite many investigations and inquiries into violent incidents in mental health settings, safety considerations for mental health staff continue to be an issue of serious concern.

Aggression and violence are often preventable. Prevention relies heavily on the awareness and skills of mental health clinicians working in organisations that are supportive and that help professionals to develop practical skills in a safe physical environment. However, there are still serious gaps.

This report replaces Safety for Trainees in Psychiatry (CR78), published in 1999. The scope of the report has been broadened to include all practising psychiatrists. The working party has reviewed the safety literature published since the last report, and consulted widely with other organisations which have similar concerns.

The report focuses on those measures which can reduce the chances of assaults taking place, or of serious injury being sustained should they occur. Trusts and other employing organisations already have statutory obligations to ensure that the environment in which mental health professionals work is safe and secure, and this report does not dwell on this area, other than to point out where psychiatrists still notice deficiencies.

Brief vignettes exemplify situations which are likely to give rise to safety considerations in various settings, and advice is given on prevention and ways to reduce escalation of risk.

Recommendations:

1. Although junior psychiatrists are now more likely to receive training in the recognition and prevention of violence, and in using de-escalation strategies and breakaway techniques, there is less evidence to suggest this is happening with senior psychiatrists. This should take place early on in the post, with regular refreshers.

2. Senior clinicians have a central role in preventing seriously aggressive behaviour in their patients. They not only have experience of assessing the risks posed by patients, but they can also advise their colleagues on how to manage such risks. Early detection of possible eruption of violence in an in-patient setting can be enhanced by a close working relationship with nurses, ability to read and take action when difficult atmospheres exist, and more active participation of psychiatrists in therapeutic activities.

3. Psychiatrists need to be aware of the potential for aggressive behaviour which might emanate as a direct result of their intervention, particularly where there exists an atmosphere of perceived confrontation, such as assessments under the Mental Health Act 1983 meetings with advocates, tribunal hearings, or where they are unrealistically expected to resolve problems outside their brief, skills or capacity.

4. Recognition of issues that require sensitive handling, in particular regarding racial or gender issues, and the careful use of language in heated interchange, can help prevent violent outbursts. The judicious use of interpreters can also calm distressed patients who are unable to communicate in English.

5. In out-patient settings, accident and emergency departments and prison environments robust safety measures need to be in place, such as adequate and well functioning alarm systems, clear exit facilities, and arrangements for support by other staff in cases of emergency. This procedure should be checked regularly. Recognition of the anxiety experienced by patients when coming to see a psychiatrist, or waiting to be seen, can do much to reduce tension and enhance the quality of the interview.

6. For psychiatrists working in the community it is important to gather as much information as possible before the visit about the patient, the family, and the environment where the visit will take place in order to take adequate preventative measures. In particular psychiatrists should carry a means of communication and ensure that the base is aware of the time of the appointment, and what to do if the psychiatrist fails to return to base within an agreed time. It is recommended that psychiatrists should not visit private dwellings alone if they are not sure about the circumstances, and never at night.

7. As far as Mental Health Act Assessments in the community are concerned, a pre-visit conference with all parties concerned, including the family, police and social workers, is strongly recommended in order to plan the intervention and take appropriate measures.

8. For those working with families and carers, in particular in old age and child and adolescent psychiatry, it is important to bear in mind that members of the family can also become highly agitated, aggressive and violent on behalf of their ill relative.

9. Psychiatrists should receive specific training on the assessment, management and risk assessments of patients with dual diagnoses

10. The working party has endorsed the recommendations of the earlier College report on Safety forTrainees (CR78), many of which apply to all psychiatrists. In particular it wishes to highlight the importance of induction courses which take into account local safety considerations, matters of personal privacy, behaviour and appearance, and clear guidelines on what must be done following a violent incident.

11. A jointly agreed and understood protocol for the reporting of untoward incidents should be in place in each workplace. This will only work if the culture allows staff to feel comfortable about reporting incidents without prejudice. Reporting incidents should be linked to a structure that allows learning to take place, and adaptation of prac tices as a result of incidents. Clinical governance principles should be followed, with regular audits of violent incidents, and effective measures introduced resulting from their recommendations.

12. In cases of serious assault, the matter should be reported to the police, who jointly with the trust should consider pressing charges against the perpetrator. In less serious incidents, a judgement needs to be made on clinical grounds whether this is the best form of action to prevent recurrences. This may need to be discussed with the legal advisor of the trust, as well as the medical defence society. 